\title{
A METHODOLOGY TO VALIDATE 3D ARBITRARY LAGRANGIAN EULERIAN CODES WITH APPLICATIONS TO ALEGRA
}

\author{
L. C. CHHABILDAS, C. H. KONRAD, D. A. MOSHER, W. D. REINHART, \\ B. D. DUGGINS, T. G. TRUCANO, R. M. SUMMERS, J. S. PEERY \\ Sandia National Laboratories, Shock Physics Applications Department, \\ Albuquerque, New Mexico 87185-1181
}

\begin{abstract}
Summary - In this study we provided an experimental test bed for validating features of the Arbitrary Lagrangian Eulerian Grid for Research Applications (ALEGRA) code over a broad range of strain rates with overlapping diagnostics that encompass the multiple responses. A unique feature of the ALEGRA code is that it allows simultaneous computational treatment, within one code, of a wide range of strain-rates varying from hydrodynamic to structural conditions. This range encompasses strain rates characteristic of shock-wave propagation $\left(10^{7} / \mathrm{s}\right)$ and those characteristics of structural response $\left(10^{2} / \mathrm{s}\right)$. Most previous code validation experimental studies, however, have been restricted to simulating or investigating a single strain-rate regime. What is new and different in this investigation is that we have performed well-controlled and well-instrumented experiments, which capture features relevant to both hydrodynamic and structural response in a single experiment. Aluminum was chosen for use in this study because it is a well-characterized material. The current experiments span strain rate regimes of over $10^{7} / \mathrm{s}$ to less than $10^{2} / \mathrm{s}$ in a single experiment. The input conditions were extremely well defined. Velocity interferometers were used to record the high strain-rate response, while low strain rate data were collected using strain gauges. Although the current tests were conducted at a nominal velocity of $\sim 1.5 \mathrm{~km} / \mathrm{s}$, it is the test methodology that is being emphasized herein. Results of a three-dimensional experiment are also presented.
\end{abstract}

\section{INTRODUCTION}

Sandia National Laboratories is developing a code referred to as ALEGRA which is a multimaterial arbitrary Lagrangian Eulerian code [1] for use in many programs related to research applications. A unique feature of ALEGRA is that it allows simultaneous computational treatment, within one code, of a wide range of strain-rates varying from encompasses strain rates characteristic of shock-wave propagation $\left(10^{7} / \mathrm{s}\right)$ and those characteristics of structural response $\left(10^{2} / \mathrm{s}\right)$ [2]. It combines the features of modern Eulerian codes such as CTH [3] with modern Lagrangian shock wave physics codes and transient structural analysis codes. Some examples of applications with varying strain rates include (but are not limited to) high velocity impact and penetration processes [4], explosively formed projectiles, and shaped charge jet formation.

Validating a code requires both postdating and predicting pertinent experimental data. The most useful validation experiments are reproducible and highly instrumented [5], with wellunderstood experimental errors. There are many parts of a calculation that we must validate: geometry, initial conditions, boundary conditions, material flow algorithms (remeshing and remapping algorithms), and material models, including EOS, constitutive relations and fracture models. There are also issues associated with meshing resolution and geometric fidelity. In many cases pertinent experimental data are available for a single strain rate regime and are used when appropriate to this regime. However, within certain applications that ALEGRA is 


\section{DISCLAIMER}

This report was prepared as an account of work sponsored by an agency of the United States Government. Neither the United States Government nor any agency thereof, nor any of their employees, make any warranty, express or implied, or assumes any legal liability or responsibility for the accuracy, completeness, or usefulness of any information, apparatus, product, or process disclosed, or represents that its use would not infringe privately owned rights. Reference herein to any specific commercial product, process, or service by trade name, trademark, manufacturer, or otherwise does not necessarily constitute or imply its endorsement, recommendation, or favoring by the United States Government or any agency thereof. The views and opinions of authors expressed herein do not necessarily state or reflect those of the United States Government or any agency thereof. 


\section{DISCLAIMER}

Portions of this document may be illegible in electronic image products. Images are produced from the best available original document. 
addressing, there is a need to perform well-controlled experiments that capture material response at both high and intermediate strain rate regimes.

In this study, we provided an experimental test bed and a methodology for validating features of the ALEGRA code [1], including material models, over a broad range of responses with overlapping diagnostics that encompass multiple strain rates. Aluminum was used in these experiments because it is a well-characterized material - its equation of state (EOS) and constitutive properties are well established over a wide range of loading rates. Pretest calculations were performed to design and optimize the experiment and to assist in instrumenting the experiment. Velocity interferometers were used to record the high strain-rate response and to determine the input conditions extremely accurately, while low strain rate data were collected using strain gauges. In particular, the current experiments span strain rate regimes of over $10^{7} / \mathrm{s}$ to less than $10^{2} / \mathrm{s}$ in a single experiment. Even though the experiments are conducted at impact velocities of $\sim 1.5 \mathrm{~km} / \mathrm{s}$, what is significant in this investigation is the methodology that is being established to validate modern 3-D Arbitrary Lagrangian Eulerian (ALE) codes, and can be easily extended to high impact velocities. The test methodology developed for use in this investigation is described in the next section. Results of these experiments including a wellcontrolled three-dimensional experiment are discussed and compared with ALEGRA simulations in subsequent sections.

\section{EXPERIMENTS}

\section{Methodology}

A series of experiments were conducted on the Sandia terminal ballistics facility [6]. This is a two-stage light-gas gun that can launch a sabot package carrying spherical projectiles to velocities over $6 \mathrm{~km} / \mathrm{s}$. A $9.52 \mathrm{~mm}, 6061-\mathrm{T} 6$ aluminum sphere was launched to $\sim 1.5 \mathrm{~km} / \mathrm{s}$. The exact impact velocity for each experiment is given in Table 1 . The impact velocity in each experiment was determined to an accuracy of $0.2 \%$ using a magnetic pick-up coil method [7]. The spherical projectile impacted one end of a hollow cylindrical can (also made of 6061-T6 aluminum) whose outer diameter was $\sim 63.5 \mathrm{~mm}$, inner diameter was $\sim 57.2 \mathrm{~mm}$, axial length was $\sim 90 \mathrm{~mm}$, with a front plate thickness of $\sim 14 \mathrm{~mm}$. The thicknesses of the front plate and the cylinder wall for each experiment is shown in Table 1. In this study, the impact velocity and the front plate thickness was controlled to prevent rupture of the plate, while causing sufficient deformation/bulging as a result of impact.

Table 1. Summary of experimental conditions

\begin{tabular}{ccccccc}
\hline $\begin{array}{c}\text { Experiment } \\
\text { Nos. }\end{array}$ & $\begin{array}{c}\text { Impact } \\
\text { Velocity } \\
(\mathrm{km} / \mathrm{s})\end{array}$ & $\begin{array}{c}\text { Front Plate } \\
\text { Thickness } \\
(\mathrm{mm})\end{array}$ & $\begin{array}{c}\text { Wall } \\
\text { Thickness } \\
(\mathrm{mm})\end{array}$ & $\begin{array}{c}\text { Impact } \\
\text { Location } \\
(\mathrm{X}, \mathrm{Y} \mathrm{mm})\end{array}$ & $\begin{array}{c}\text { Radius } \\
(\mathrm{mm})\end{array}$ & $\begin{array}{c}\mathrm{x}-\mathrm{t} \\
\text { Slope }\end{array}$ \\
\hline L1 & 1.48 & 13.614 & 3.18 & $+0.12,+2.5$ & 2.502 & 5.82 \\
L2 & 1.52 & 13.919 & 3.18 & $+2.5,+2.5$ & 3.535 & 5.59 \\
L3 & 1.47 & 13.919 & 3.20 & $+11.4,-3.8$ & 12.016 & 5.73 \\
L4 & 1.55 & 14.021 & 3.23 & $-3.8,+4.3$ & 5.738 & 5.57 \\
L5 & 1.52 & 13.665 & 3.19 & $-2.0,+5.2$ & 5.571 & 5.66 \\
L6 & 1.52 & 13.614 & 3.23 & $-2.0,+3.0$ & 3.606 & 5.66 \\
\hline
\end{tabular}


The experimental configuration is indicated in Figure 1(a). A velocity interferometer, VISAR, [8] is used to monitor the back surface motion of the free surface both along the central axis and at off-axis locations. A total of twelve strain gauges, six to determine the axial strain (stress) and six to determine the hoop strain (stress), were used. The measurements were determined to an accuracy of better than $2 \%$ for velocity histories and $3 \%$ for strain gauge records. In experiments L1 and L2, two polyvinyldifluoride (PVDF) gauges were also used on the circumferential surface but orthogonal to the strain gauge records. Because the output signals from the PVDF gauges perturbed the strain gauge records they were no longer used in subsequent experiments. In subsequent experiments they were replaced with strain gauges. Figure 1(a) illustrates the location of the velocity interferometer, and the strain gauges used in this study. An instrument can assembly is shown in Figure 1(b). Strain gauges 1 to 3 are positioned on one side of the can while gauges 4 to 6 are installed diametrically to strain gauges 1 to 3 . The strain gauges are positioned nominally at $19 \mathrm{~mm}$ (gauges $1 \& 4$ ), $48 \mathrm{~mm}$ (gauges $2 \&$ 5 ) and $78 \mathrm{~mm}$ (gauges $3 \& 6$ ) from the impact plane, and along the circumferential surface of the cylinder.

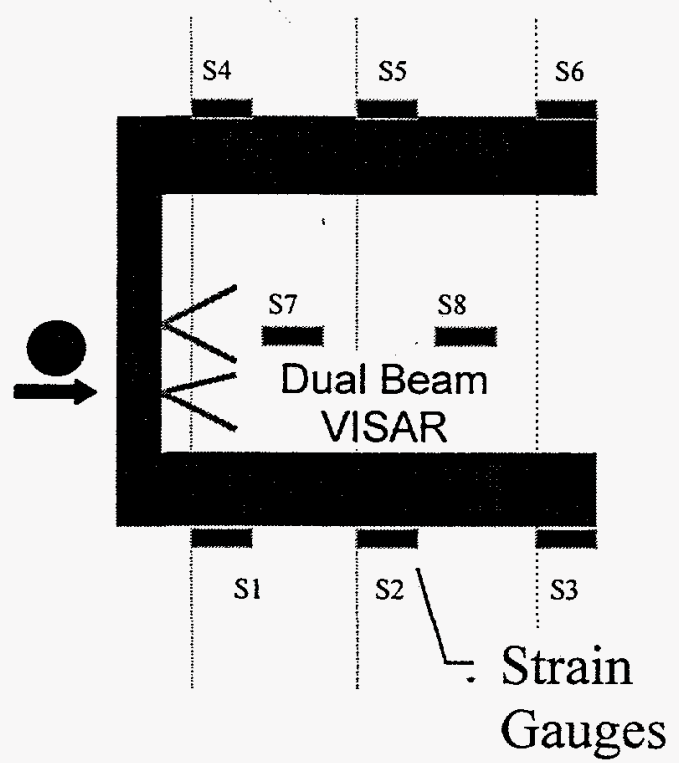

(a)

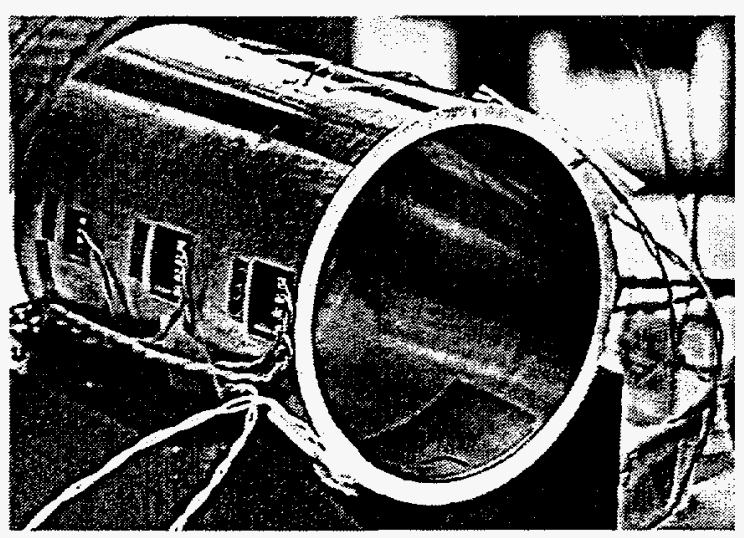

(b)

Fig. 1. Experimental configuration is shown in (a), while the instrumented can is shown in (b).

The measured free-surface particle velocity history is shown in Figure 2 for all the experiments. The strain gauge records, which represent the axial strain measurements of strain gauges 1 to 3, and 4 to 6 along the circumference are indicated in Figures 3 and 4, respectively, for experiment L5. The arrival time of the stress front as indicated by the strain gauge records is plotted versus its location in Figure 5. (In this graph, the times are arbitrarily shifted to allow a display of all experiments.) This yields the rate at which the stress front sweeps up in the cylindrical tube. As indicated in Table 1, multiple experiments were performed to determine the accuracy, and the repeatability of the experiments. The impact velocity was reproducible to within $1.3 \%$ of the mean impact velocity of $1.5 \mathrm{~km} / \mathrm{s}$. All impact locations (except L3) are within $2.5 \mathrm{~mm}$ and $5.7 \mathrm{~mm}$ from the geometric center of the instrumented can, and are well within half the projectile sphere diameter. 


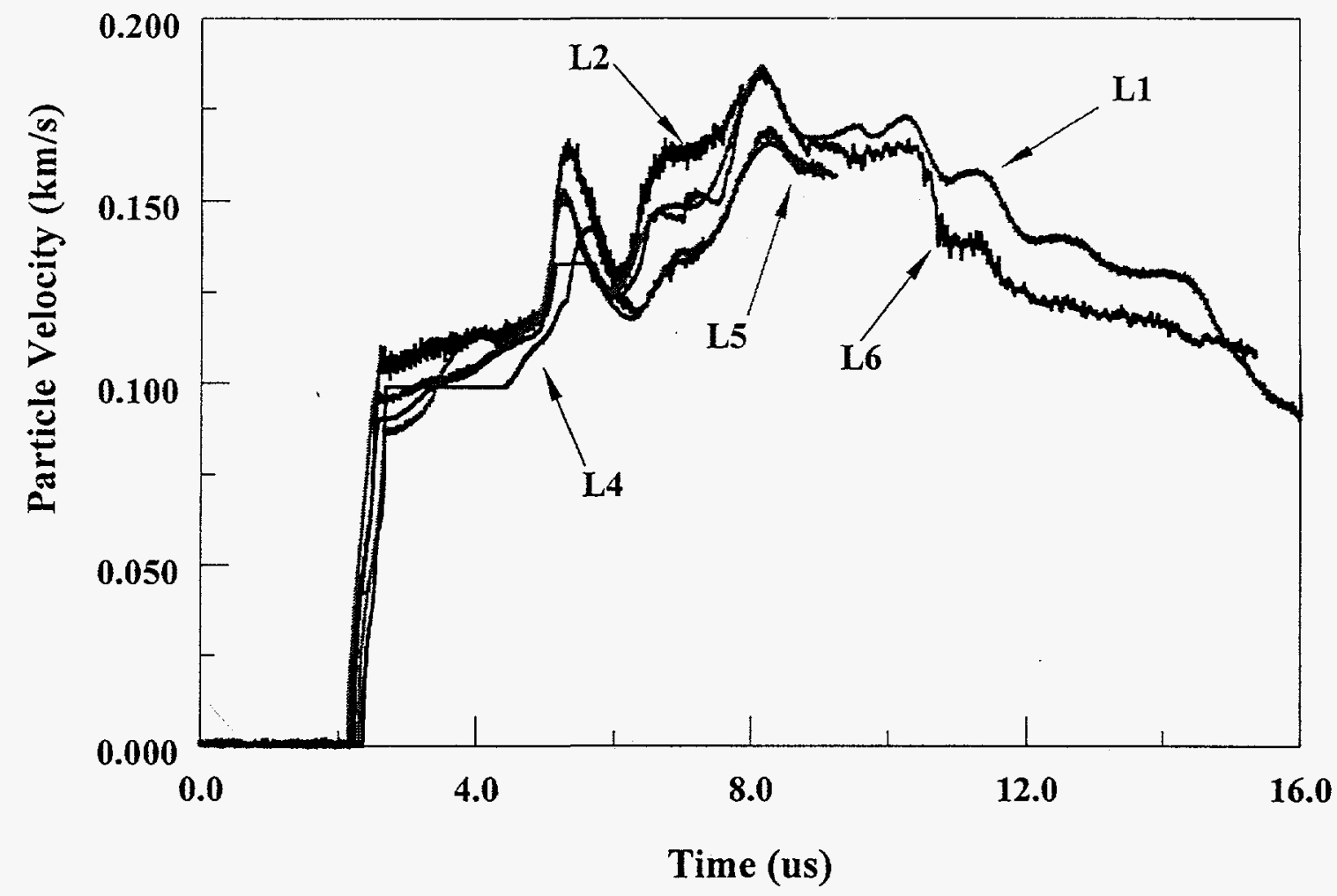

Fig. 2. Free-surface velocity time history of back surface motion along the central axis of the can.

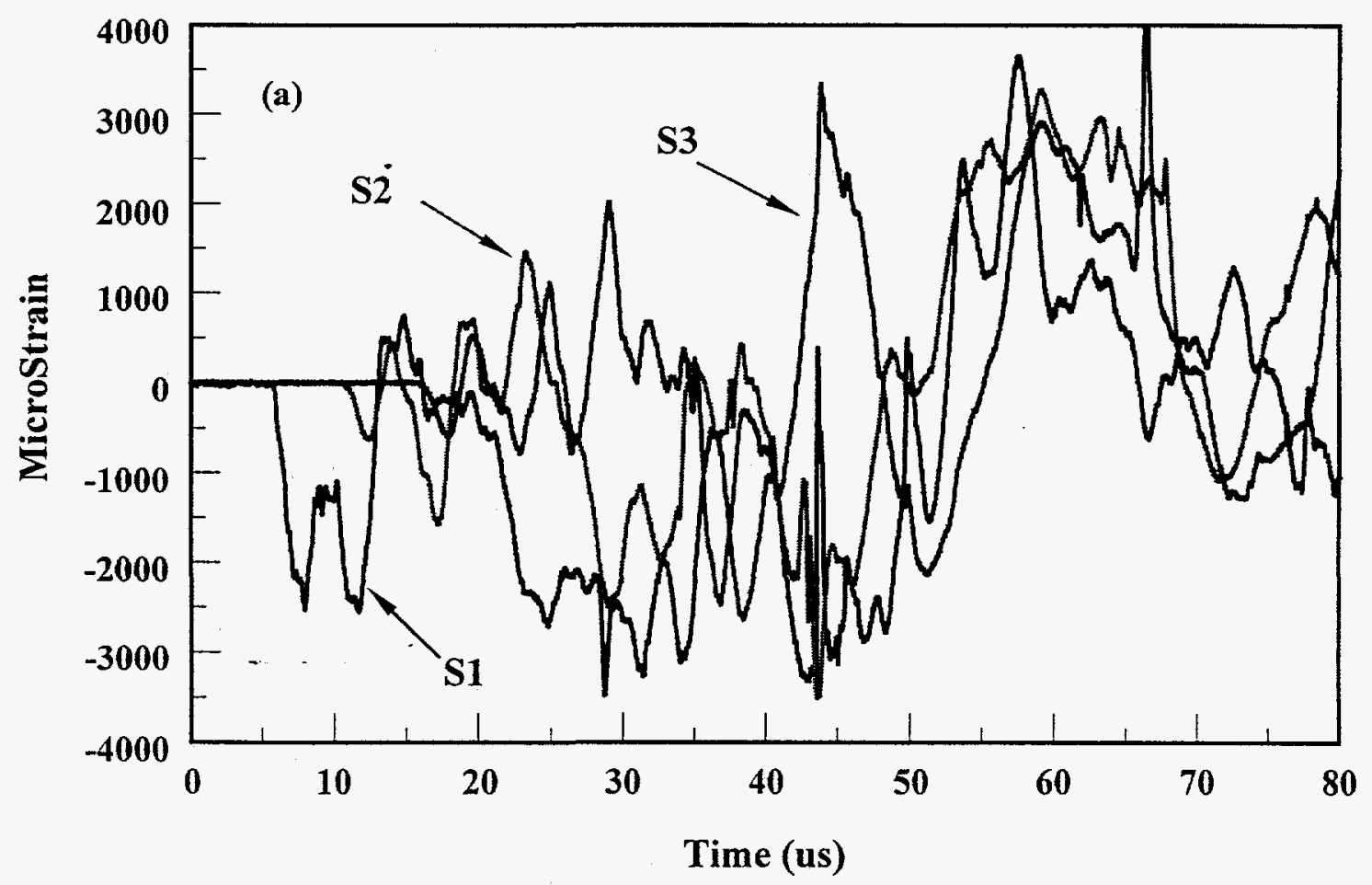

Fig. 3. Axial strain gauge records 1,2 , and 3 for experiment $L$. 


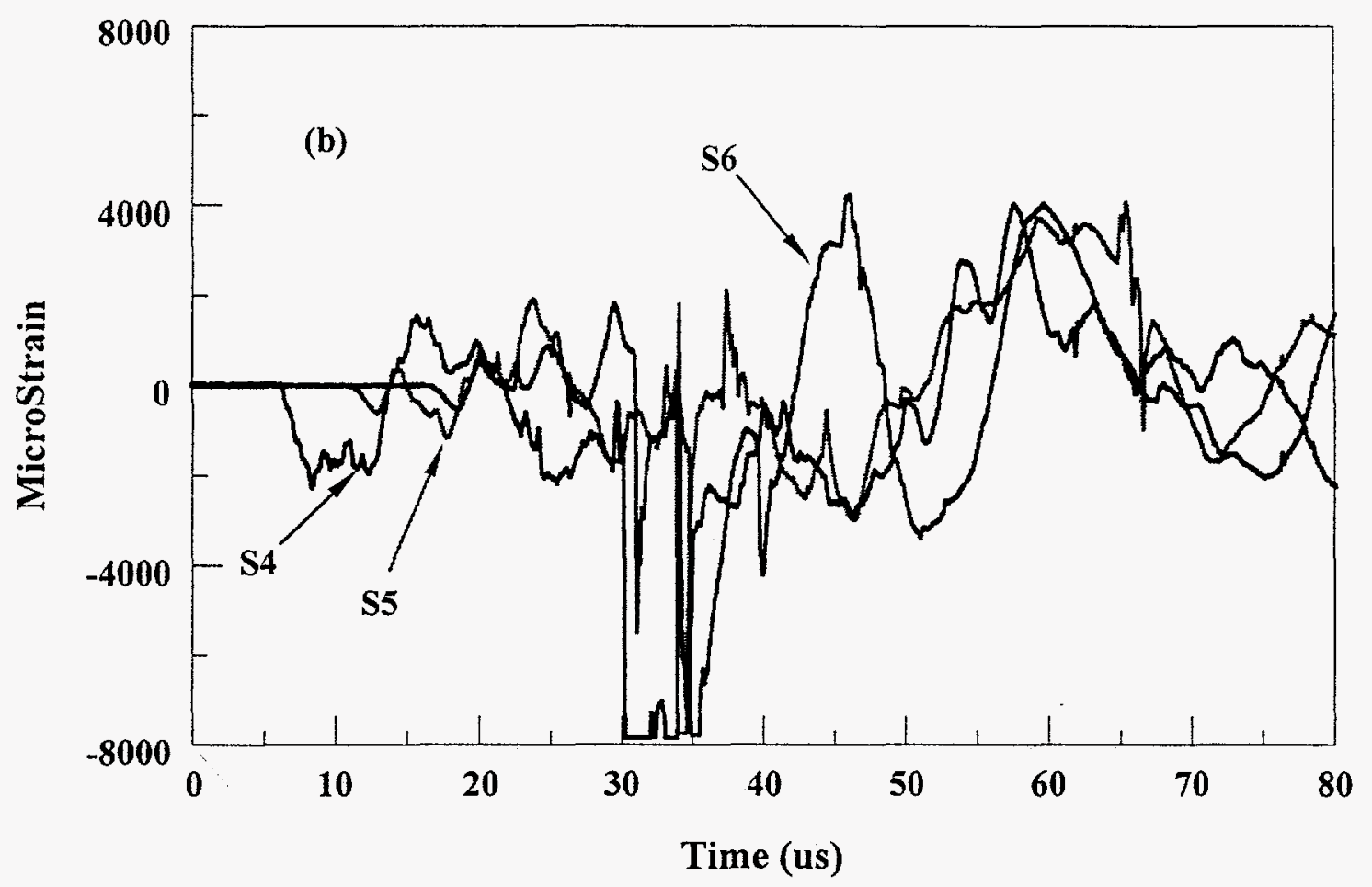

Fig. 4. Axial strain gauge records 4, 5, and 6 for experiment L5.

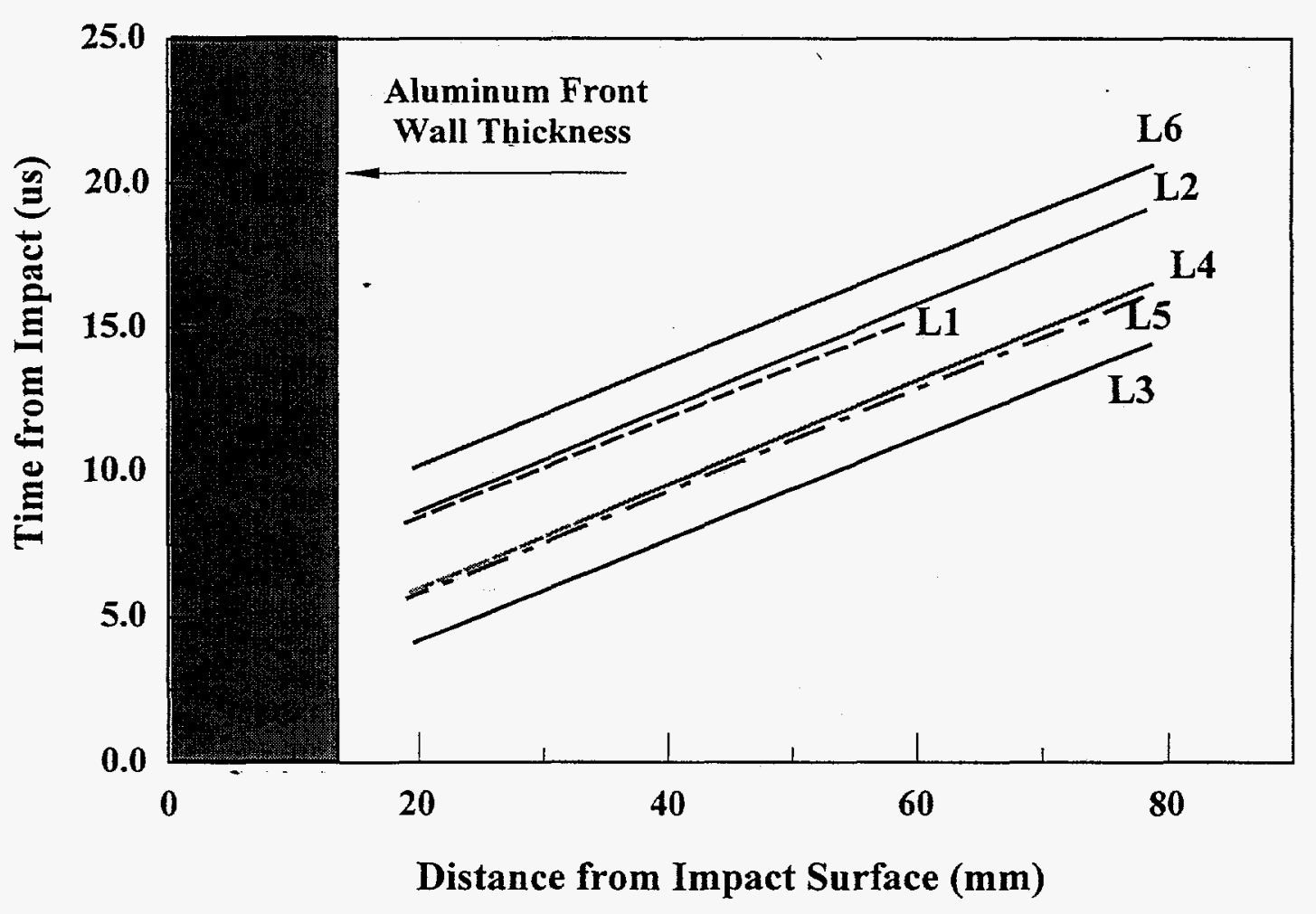

Fig. 5. x,t diagram of axial gauge records for all experiments. The slope of the lines represents the speed of the wave propagation front (see Table 1). 
For the purposes of discussion in this paper these experiments are characterized as twodimensional. This potentially allows a detailed analysis to capture the effects of slight variations in experiments while assuming a two-dimensional axis symmetric configuration for computational analysis. Note the deviation is small considering that the sphere is launched over a distance of 6 meters from the muzzle of the gun to the impact location.) In experiment L3, the sphere impacted the front face of the aluminum can at a radius of $\sim 12 \mathrm{~mm}$ from the center, i.e., the axis of symmetry. This is clearly over one ball diameter; the results of this experiment will address three -dimensional impact effects both experimentally and computationally.

\section{Results}

Two-Dimensional (2D) Experiments - VISAR Records. Impact locations are within $2.5 \mathrm{~mm}$ and $5.7 \mathrm{~mm}$ from the geometric center of the instrumented can, and are well within half the projectile sphere diameter. Upon impact, peak stresses approaching $13 \mathrm{GPa}$ are generated at the contact point. The loading strain rates at that point are in excess of $10^{7} / \mathrm{s}$. A spherical diverging wave, in combination with edge relief, attenuates the resulting stress wave. The peak velocity measurement of approximately $0.2 \mathrm{~km} / \mathrm{s}$ at the rear free surface for all the experiments indicates substantial wave attenuation. The velocity interferometer was set up to monitor the particlevelocity at the exact geometric center of the can. The variation in free-surface particle velocities in Figure 2 results from variation in impact velocity and the impact location. These results, therefore, would allow an estimate of the dispersion/attenuation of the wave propagation process occurring radially over the impact locations of $2 \mathrm{~mm}$ to $5 \mathrm{~mm}$ from the center axis. The leading edge of the wave in the front plate is determined to travel at $6.4 \mathrm{~km} / \mathrm{s}$, which is representative of the elastic wave velocity in 6061-T6 aluminum. Although not shown in this paper, the off-axis velocity interferometer measurements also suggest that the initial arrival time of the diverging stress wave is indicative of an elastic wave front. The leading precursor wave velocity is determined to an accuracy of $1 \%$.

Two-Dimensional (2D) Experiments - Strain Gauge Records. In these experiments, the relative time of arrival of all strain gauge records are known to within the sampling rate of the recording equipment, which is $20 \mathrm{~ns}$ for the current strain gauge records. The strain gauge records for experiment L5 are indicated for gauges 1 to 3 in Figure 3 and for gauges 4 to 6 in Figure 4. Not all experiments are indicated in this paper but they have been documented elsewhere (9). Gauge records indicate peak strain of $\sim 2500 \times 10^{-6}$ at a strain rate of $1.2 \times 10^{3} / \mathrm{s}$ at approximately $20 \mathrm{~mm}$ from the impact interface. This reduces to a strain of $500 \times 10^{-6}$ at a strain rate of $2 \times 10^{2} / \mathrm{s}$ at about $80 \mathrm{~mm}$ from the impact interface.

$X-t$ Diagram. Figure 5 shows the least squares fit lines to the first arrival time of the strain gauge record versus its location for all the strain gauges used in the experiment. All experiments are shown in Figure 5. The initial arrival time is intentionally shifted arbitrarily to display the slopes of the lines for all experiments. The gauge pairs $\{1,4\},\{2,5\}$, and $\{3,6\}$ are located at the same location but diametrically opposite from the impact interface on the circumferential surface. Experimentally, however, the arrival time of the leading edge of the wave is different for the complementary gauge (See Figure 3 and Figure 4). The time difference between the two gauges at the same location but diametrically opposite to each other is due to the non-centered nature of the impact. The data indicates that the stress front sweeps by at an average velocity of $5.66 \mathrm{~km} / \mathrm{s}$ in the cylindrical tube. Thus, results of the strain-gauge records versus location in each experiment yield a velocity that is independent of the minor variations in both impact velocity and impact location for the series of experiments reported herein. This suggests that an inclined wave front is propagating in the cylindrical tube and it travels at the same wave speed.

Three-Dimensional (3D) Experiment - VISAR Records. In experiment L3 the impact occurs at a radius of $12 \mathrm{~mm}$ from the geometric center. Peak stresses approaching $13 \mathrm{GPa}$ are generated at the contact point at impact. The loading strain rates as in other experiments is in excess of $10 \%$. A spherical diverging wave, in combination with edge relief, attenuates the resulting 
stress wave. The peak velocity measurement of $\sim 0.035 \mathrm{~km} / \mathrm{s}$ monitored at the exact geometric center of the can, shown in Figure 6, indicates significant wave attenuation for non-centered impact. The attenuation is an order of magnitude more than what is indicated for the 2D experiments in Figure 2. The leading edge of the wave in the front plate is determined to travel at $6.4 \mathrm{~km} / \mathrm{s}$ as determined by the distance from impact point to the center of the can. This is representative of the elastic wave velocity in 6061-T6 aluminum. This leading precursor wave velocity is also determined to an accuracy of $1 \%$ in this experiment.

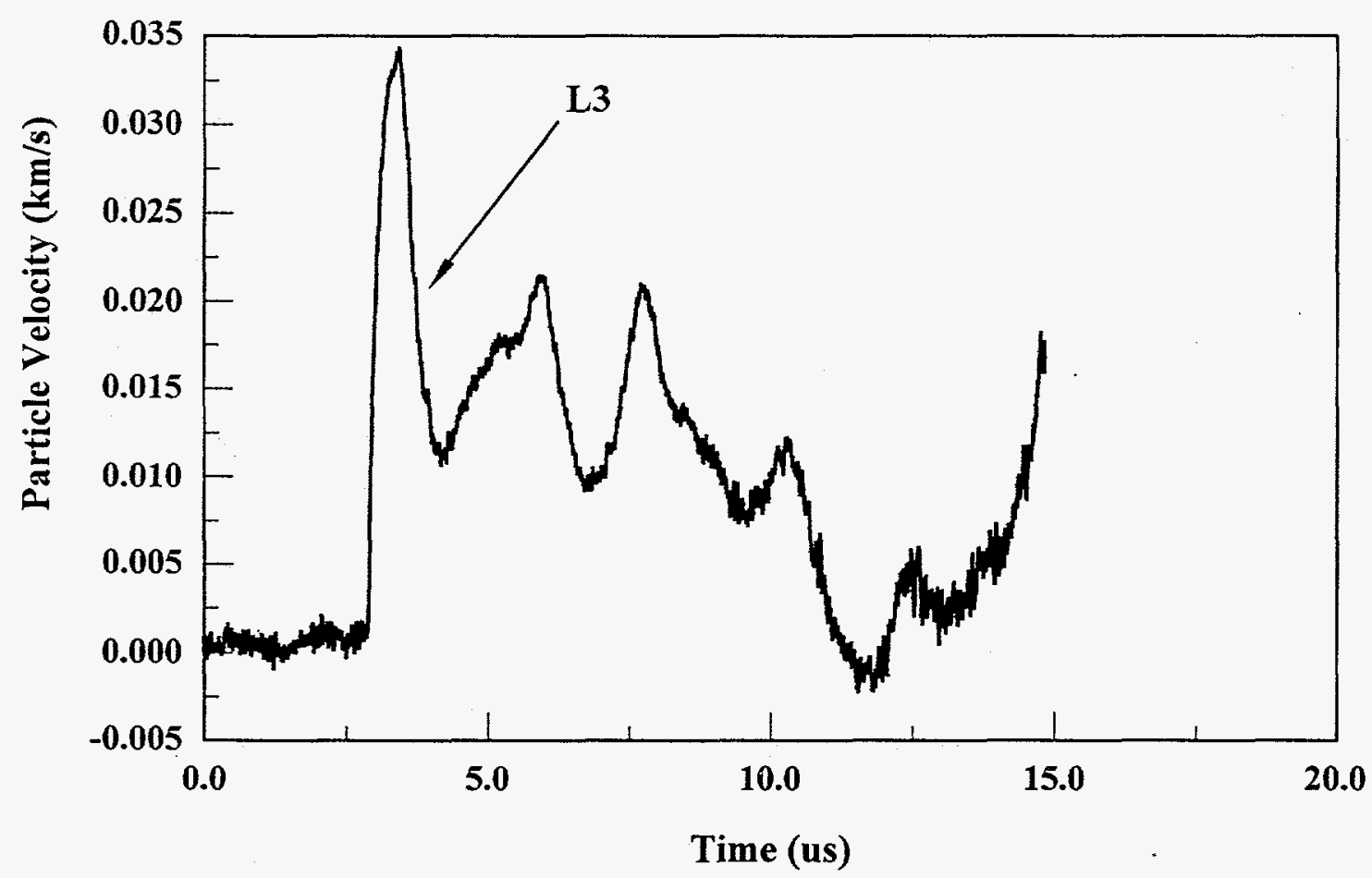

Fig. 6. Free-surface velocity time history of back surface motion along the central axis of can.L3. Impact occurred at a radius of $12 \mathrm{~mm}$ from center.

Three-Dimensional (3D) Experiment - Strain Gauge Records. The strain gauge records for experiment L3 are shown for gauges 1 and 2 in Figure 7 and for gauges 4 to 6 in Figure 8 . The records indicate peak strain of over $8000 \times 10^{-6}$ at a strain rate of $1.2 \times 10^{3} / \mathrm{s}$ at approximately 20 $\mathrm{mm}$ from the impact interface. The oscillatory behavior appears to be considerably asymmetric when compared to the strain gauge records of the two-dimensional L5 experiment in Figures 3 and 4.

$X-t$ Diagram. Figure 5 shows the least squares fit line to the first arrival time of the strain gauge record versus gauge location for gauges 4 to 6 in experiment L3 (Only 4, 5, and 6 were used because we did not get a gauge record 3 for the companion gauge 6). All other experiments are also shown in Figure 5. As shown in Figures 7 and 8, the arrival time of the leading edge of the wave is approximately 3.5 us different for the complementary gauges 1 and 4 . This large time difference between the two gauges at the same location but diametrically opposite to each other is evidently due to the 3D aspect of the experiment. The data nevertheless indicates that the stress front sweeps by at a velocity of $5.73 \mathrm{~km} / \mathrm{s}$ in the cylindrical tube, and is quite comparable to the mean velocity of $5.66 \mathrm{~km} / \mathrm{s}$ observed in the $2 \mathrm{D}$ experiments. The large time differences between the complementary gauge records suggest considerable obliquity in the $3 \mathrm{D}$ experiment. Incidentally, this wave speed is very close to the estimates of theoretical plate velocity in 6061 T6 aluminum, which is about $5.60 \mathrm{~km} / \mathrm{s}$. 


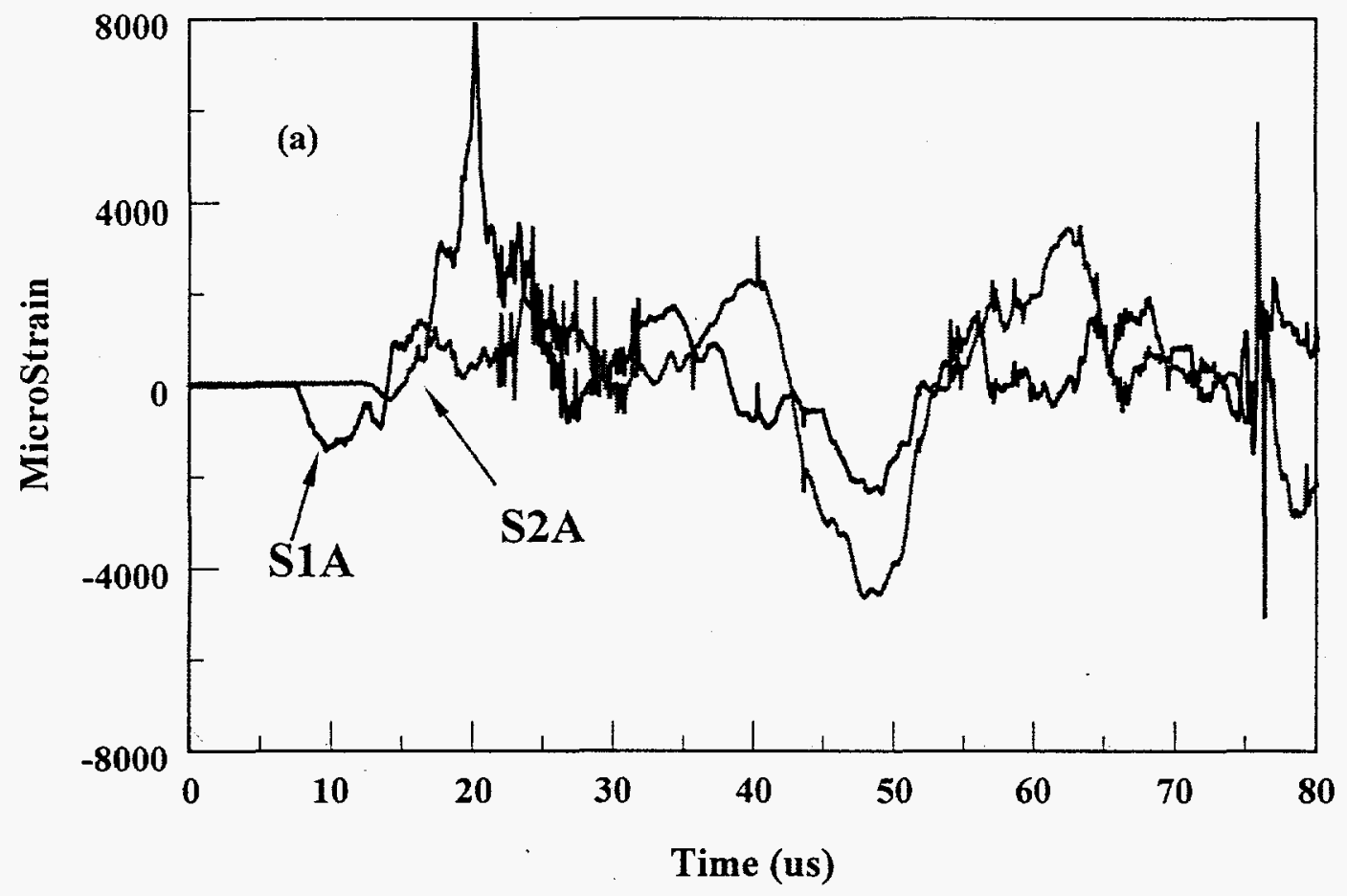

Fig. 7. Axial strain gauge records 1 and 2 for experiment L3.

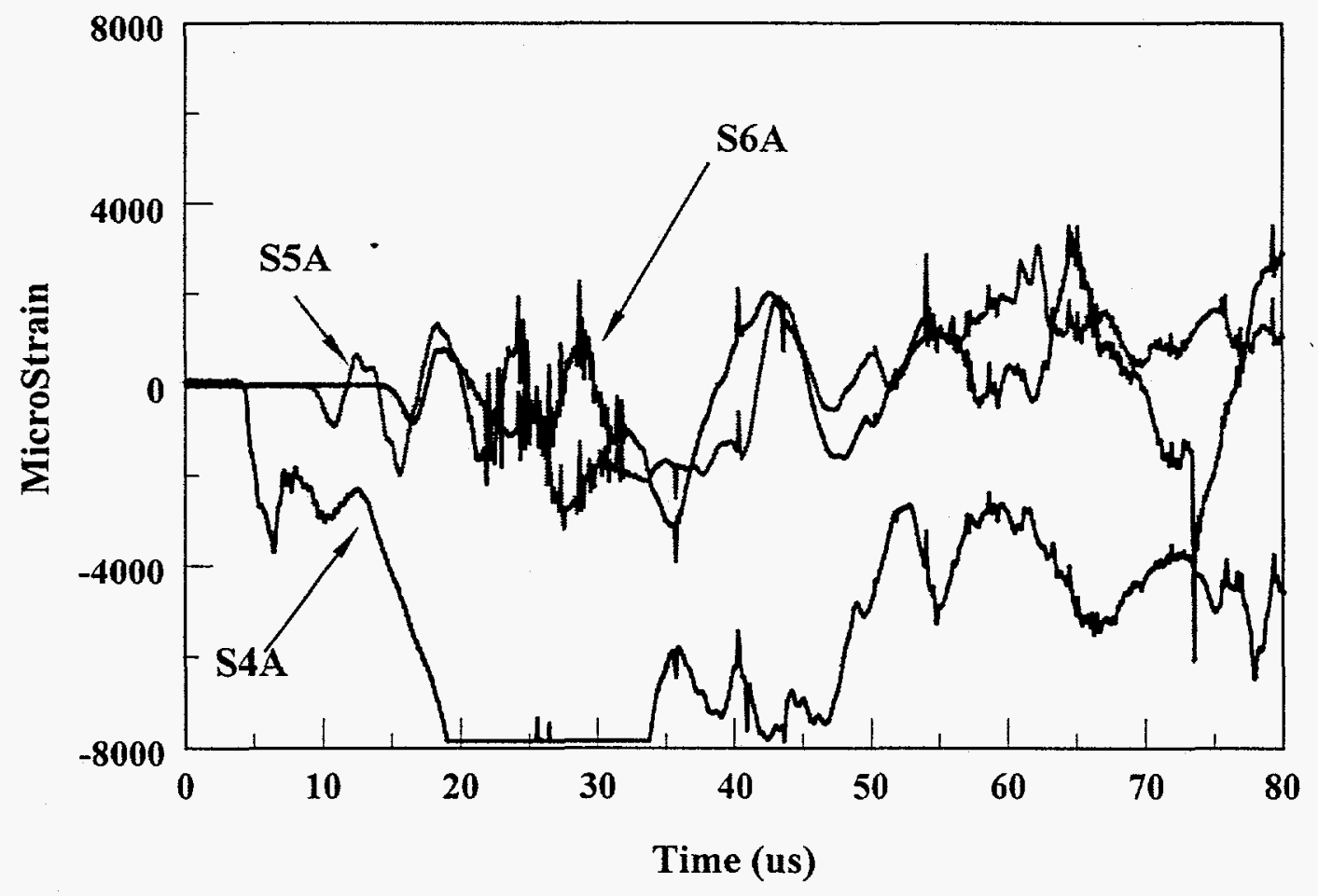

Fig. 8. Axial strain gauge records 4, 5, and 6 for experiment L3. 
Degree of Asymmetry. Table 1 summarizes the location of impact both as an $\mathrm{x}, \mathrm{y}$ coordinate and also as the radius measured from the center of the can, which is regarded as the origin. The degree of asymmetry is defined as the difference between the lines $\left(R R_{1}\right)$ and $\left(R R_{4}\right)$. $R$ is the location of the impact (see Table 1), and the coordinates for $R_{1}$ and $R_{4}$ are $(-31.6,0)$ and $(31.6$, 0 ), respectively, the edges of the front surface where gauges $1,2,3$ and $4,5,6$ are installed. These lines are shown schematically in Figure 9. Note, if impact occurs along the y-axis then the impact location is symmetric with respect to the gauges located diametrically opposite to each other. There will not be any asymmetry with respect to the arrival times of the stress front at the gauge locations - even though the experiment may be a 3-D experiment. The time difference between the two gauges at the same location but diametrical to each other is primarily due to the asymmetry, and not merely due to the non-centered nature of the impact. The experiment L3 is, therefore, not only a 3D experiment, but from the viewpoint of strain-gauge locations, an asymmetric experiment. The degree of asymmetry is plotted versus the time difference between the two strain gauges at the same location in Figure 9. There appears to be a systematic correlation as indicated in the figure. Also shown in the figure are photos of recovered cans displaying the impact location in each experiment. From the stand point of code validation and computational analysis experiment $\mathrm{L} 1$ is the best experiment to investigate axis symmetric 2D effects, while experiment L 3 is the best asymmetric 3D-experiment. The results of experiment L2 are not indicated in Figure 9. This is because good strain gauge records were not recorded in experiment L2.

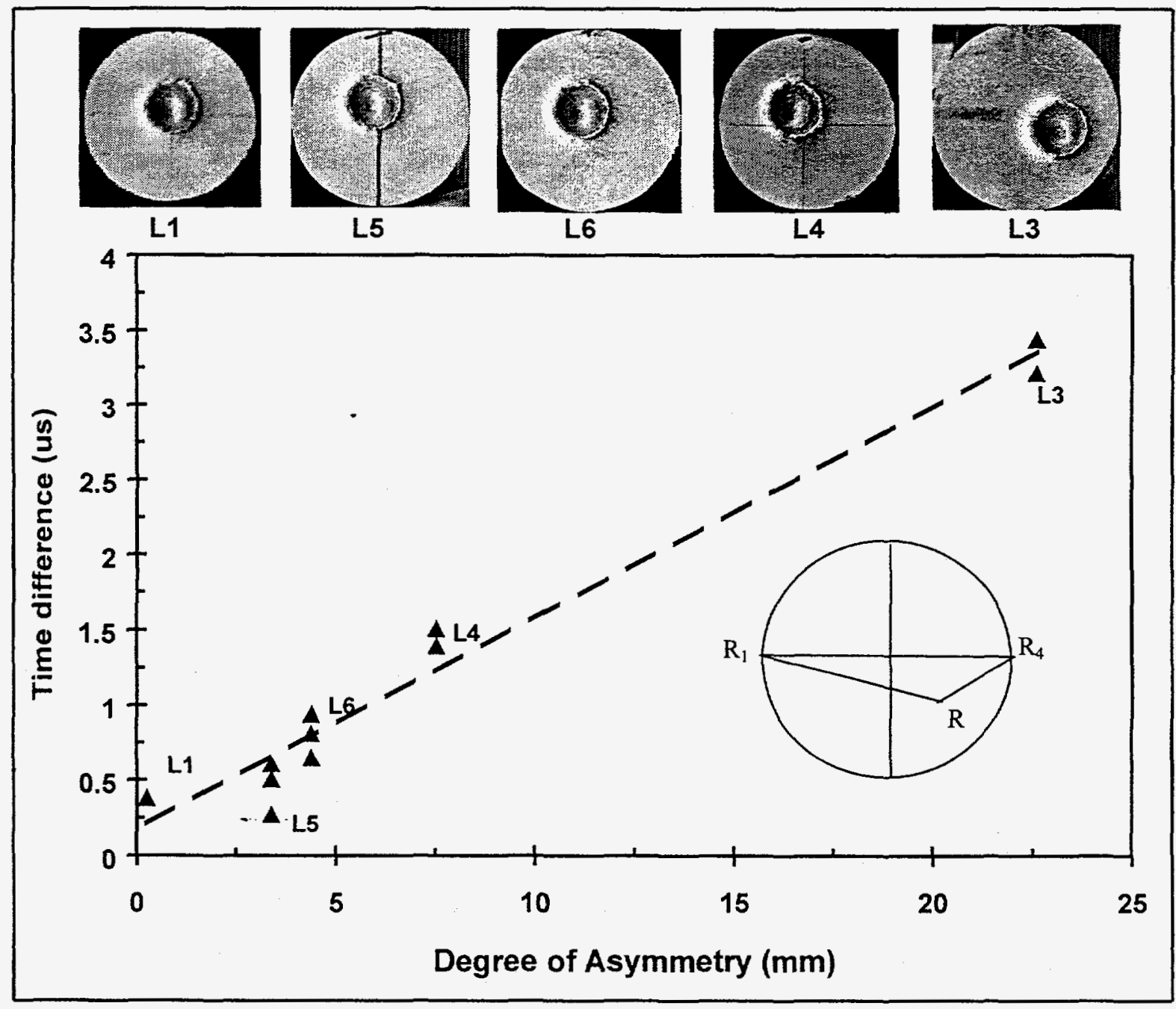

Fig. 9. Degree of asymmetry versus time difference between opposing strain gauges. Degree of asymmetry is defined as the difference in lines $\mathrm{RR}_{1}$ and $\mathrm{RR}_{4}$. 
The reasons for conducting the ALEGRA simulations are three-fold: 1) to assist in the design of the validation experiments; 2) to produce results for comparison with the experimental data; and 3) to utilize the code and discover errors and inadequacies from a user's perspective. The combined goal is ultimately to contribute to the validation of the ALEGRA code for a certain class of problems or determine the net uncertainty from various possible sources of error. Since there is always the desire to improve the accuracy of a code, an additional goal is also to discriminate between the dominant individual sources of error.

Axi-symmetric two-dimensional simulations with Eulerian meshes were run in the baseline studies. A Mie-Gruneisen EOS and an elastic perfectly plastic constitutive relation were used for simplicity. In the experiments, velocities at the centerline were measured for the off-center impacts, whereas in the two dimensional simulations this was approximated by off-center velocity measurements of centered impacts. This approximation will be most appropriate for early times (less than $20 \mu \mathrm{s}$ ) and for lower frequencies at the longer times.

Both the VISAR and strain gauge measurements were made on the material surface. Timeresolved data for Eulerian calculations in ALEGRA are acquired by the use of massless Lagrangian tracers. These tracers move with the Lagrangian motion of the materials during the course of calculations. Because of tracking problems these tracers must be placed at least one zone away from material boundaries in order to move most accurately. Otherwise, numerical diffusion associated with multi-material Eulerian interface tracking will partially corrupt the data recorded by the tracers. In our simulations, results were recorded at locations, which were 1.5 and 2.5 zones from the free surface to examine the effect of tracer location. Meshes with 0.5 , 0.25 and $0.125 \mathrm{~mm}$ cell dimensions were used to examine mesh convergence effects. For the velocity type data as shown in Figure 2, a mesh size of $0.25 \mathrm{~mm}$ produced adequate convergence to $\sim 2 \%$ error for the early time response. There was very little change in the calculated velocity history when the mesh size was further reduced to $0.125 \mathrm{~mm}$, indicating mesh convergence for these mesh dimensions.

The consistency in Figure 10 between experiment and simulation is good, especially at early times and for lower frequencies at later times. The higher frequency details at longer times are not captured well due in part to the off center effects mentioned above. Three-dimensional analyses are currently being set up to more accurately model the experiment. Based on a limited set of materials testing data, the yield strength of the aluminum was initially taken to be $300 \mathrm{MPa}$, and the early time velocity comparison

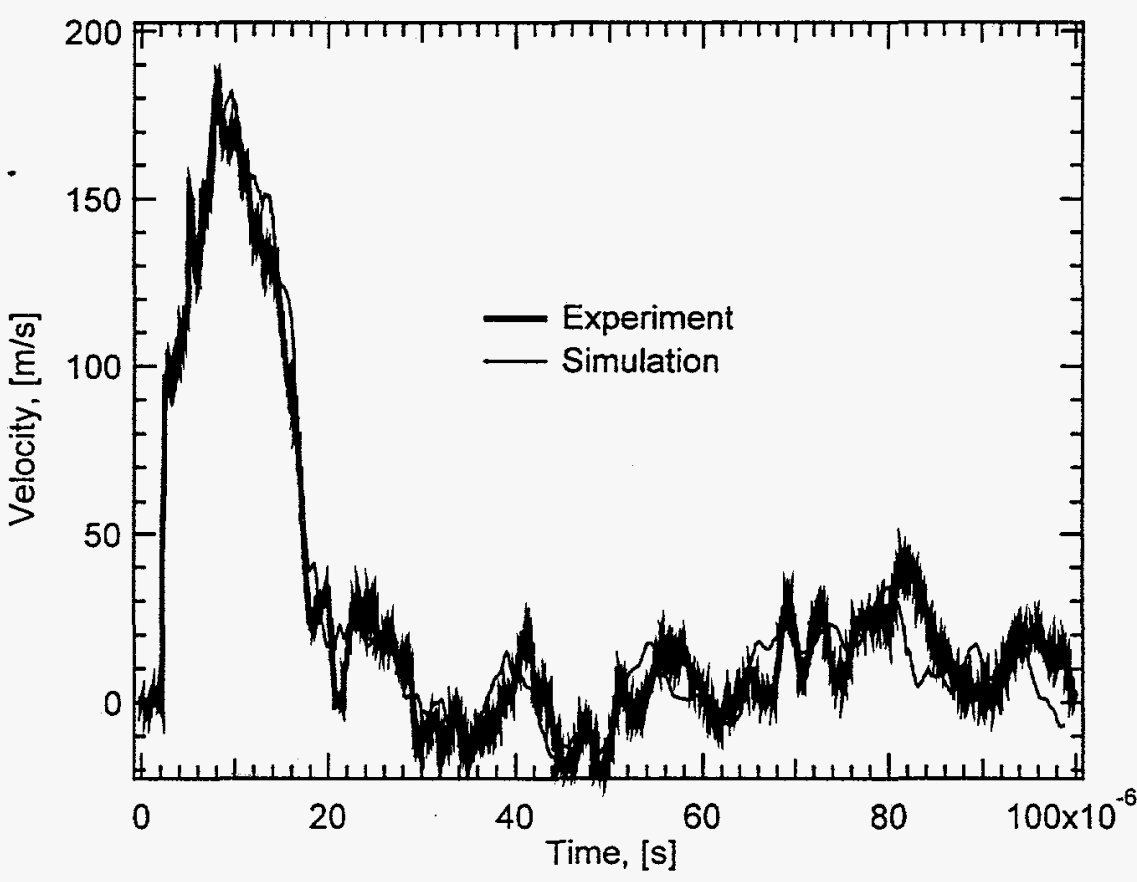

Fig. 10. Comparison of calculational simulations (indicated as faint line) of the velocity history in experiment L1 with the measured velocity history (dark line). 
- disagreement, as. shown in Figure 11. Subsequent examination of split Hopkinson bar results indicated that a choice of $400 \mathrm{MPa}$ would be more appropriate. As indicated in Figure 11, the use of this value in the calculation resulted in much better agreement.

Total strain currently is not output from the code, but the stress components are. Since yielding was not observed at the strain gauge locations in the simulations, the elastic strains were calculated from the stresses and used in comparisons with experiment. Figure 12 shows the simulation and experiment for the strain gauge location S2. Initially, the results agree reasonably well, but at later times disagreement becomes more significant. Possible causes are the accumulation of error from advection, threedimensional effects, or the effect of artificial viscosity on elastic wave propagation. The mesh size convergence of the strain gauge records did not appear to be as good as for the velocity data because long time response is desired from the former, so that there is more time for errors to accumulate. Likewise, studies with ALE meshes, in which the strain gauged side wall was Lagrangian, did exhibit slightly better agreement with experiment than pure Eulerian ones, and it is anticipated that the improvement would be greater at longer times. Further study, including three dimensional simulations, and further study of ALE approaches, is required to draw firm conclusions about the uncertainty of the code for this type of problem. 
In this study, we provide an experimental test bed and a test methodology for validating features of the ALEGRA code over a broad range of responses with overlapping diagnostics that encompass the multiple strain rates. Specifically, the current experiments span the strain rate regimes from $10^{7} / \mathrm{s}$ to less than $10^{2} / \mathrm{s}$. Input conditions are well characterized; the input conditions are known to better than $0.2 \%$, while the measurement precision is approximately $2 \%$ for the interferometer records and about $3 \%$ for the strain gauge records. The current experiments are well controlled two-dimensional and three-dimensional loading experiments.

Results of the experiments indicate that in the front thick plate the diverging wave propagates at an elastic wave-velocity of $6.4 \mathrm{~km} / \mathrm{s}$. The wave transitions into the thin cylindrical tube at an average plate wave velocity of $5.66 \mathrm{~km} / \mathrm{s}$. The strain gauges located on the tube wall suggest an asymmetry in wave propagation and can be correlated to the degree of asymmetry as defined in this paper. The data is collected over long time scales. Even though it is not displayed in this study strain gauge records have been gathered for up to a millisecond.

Future experiments will consist of well-controlled three-dimensional loading conditions. Future experiments will also include a test bed at higher impact velocities, and an increased complexity of the test bed. The cylindrical can will be filled with structural materials of interest such as foam and steel to simulate many research and structural applications. This is ongoing work and it is anticipated that the current data set will be continuously used to evaluate many aspects and issues related to ALEGRA code validation.

Acknowledgement-Technical discussions with the ALEGRA code team were extremely valuable. The authors acknowledge the enthusiastic interest in this work by J. Moya, T. Bickel, W. Kawahara, and J. Asay all of Sandia National Laboratories. Sandia is a multi-program laboratory operated by Sandia Corporation, a Lockheed Martin Company for the United States Department of Energy under contract DE-ACO4-94AL85000.

\section{REFERENCES}

1. R. M. Summers, J. S. Peery, M. W. Wong, E. S. Hertel, T. G. Trucano, L. C. Chhabildas, Recent progress in ALEGRA development and applications to ballistic impact. Int. J. Impact Engng., 20, 779-788 (1997).

2. K. G. Budge, J. S. Peery, RHALE: a MMALE shock physics code written in C++. Int. J. Impact Engng., 14, 107-120 (1993).

3. J. M. McGlaun, S. L. Thompson, M. G. Elrick, A brief description of the three-dimensional shock wave physics code CTH. Sandia National Laboratories Report SAND89-0607, (1989).

4. D. E. Carroll, E. S. Hertel, T. G. Trucano, Simulation of armor penetration by tungsten rods: ALEGRA validation report. Sandia National Laboratories Report SAND97-2765, (1997).

5. L. C. Chhabildas, Survey of diagnostic tools used in hypervelocity impact studies. Int. J. Impact Engng., 5, 734-743 (1987).

6: J. R. Asay, L. C. Chhabildas, L. M. Barker, Projectile and impactor designs for plate-impact experiments. Sandia National Laboratories Report SAND85-2009, (1989).

7. R. L. Moody, C. H. Konrad, Magnetic induction system for two-stage gun projectile velocity measurements. Sandia National Laboratories Report SAND84-0638, (1984).

8. L. M. Barker and R. E. Hollenbach, Laser interferometer for measuring high velocities for any reflecting surface. J. Appl. Phys., 43, 4669-4675.

9. L. C. Chhabildas, C. H. Konrad, D. A. Mosher, W. D. Reinhart, B. D. Duggins, T. G. Trucano, R. M. Summers, J. S. Peery, ALEGRA code validation: experiments and simulations. Sandia National Laboratories Report in preparation. 